\title{
Effective Land Surface Temperature Retrieve from Image Data Using Fast Fuzzy Random Clustering Algorithm
}

\author{
Rajkumar.C ${ }^{1}$, Binu.B ${ }^{2}$ \\ Asst. Professor, Department of Computer Science, Kaamadhenu Arts and Science College, Erode, Tamilnadu, India ${ }^{1}$ \\ M. Phil Scholar, Department of Computer Science, Kaamadhenu Arts and Science College, Erode, Tamilnadu, India ${ }^{2}$
}

\begin{abstract}
Land Surface Temperature (LST) may be a key variable in climatological and environmental studies. However, correct measurements of LST over continents aren't nevertheless on the market for the whole globe. This paper initial reviews the state of the science of land surface temperature (LST) estimates from remote sensing platforms, models, and in place approaches. In this thesis analysis a physics-based technique to retrieve LST from the MODIS daytime MIR information in channels twenty two (centered at $3.97 \mu \mathrm{m}$ ) and twenty three (centered at 4.06 $\mu \mathrm{m}$ ). On the premise is radiative transfer theory within the MIR region, a bi-face reflectivity retrieval technique. During this technique to separate the mirrored star direct irradiance and also the radiances emitted by the surface and atmosphere. MIR Data Land Cover temperature measured is asserted once consecutive sub sequences that are extracted from one MODIS statistic transitions from one cluster to a different cluster and remains within the freshly appointed cluster for the remainder of the statistic.
\end{abstract}

Keywords: LST, MIR, Fuzzy Random Clustering, Land Cover Process, Image Clustering

\section{INTRODUCTION}

The introduction of the Mid Infra Red (MIR) channels into LST retrieval will considerably cut back the correlation of the sets and greatly improve the accuracy of the calculable LST. the most objective of this thesis is to develop a physics-based methodology to retrieve Land Surface Temperature (LST) from the Moderate Resolution Imaging Spectro radiometer (MODIS) daytime MIR information. The study on LST retrieval from Mid-Infrared (MIR) information is under-developed as a result of the radiance measured throughout daytime within the MIR spectrum contains each the surface emitted thermal radiance and also the mirrored star radiance, that ar equal in magnitude. The MIR information has its own blessings, like higher detection sensitivity for top temperatures, higher atmospherical transmission within the atmospherically window and fewer sensitive to vapour content. In this thesis, the daytime MIR radiance contains not solely the radiance emitted by the land surface and atmosphere however additionally the star radiance mirrored by the land surface and scatted by the atmosphere. To retrieve LST from MIR information, the direct star radiance ought to be calculable initial to eliminate the impact of star radiance, so a split window methodology ought to be developed to estimate the LST victimization the radiance once extracting the mirrored star direct radiance. The MODIS image is obtained and also the surface kind image classification is performed during this thesis so most like hood classification is enforced with the automated image classification approach. For the classification of the land surface temperature and surface pictures, fuzzy automatic bunch formula is enforced. The planned LST retrieval formula is applied to the Moderate Resolution Imaging Spectro radiometer over MIR information and is valid over 3 land surface sorts with in place measurements.

Objective of The Proposed System

- Clustering methodology is enforced to method subsequences of your time series information.

- Detects land cowl modification temperature measured as a operate of your time.

- Land cowl modification temperature measured in consecutive time intervals.

- Subsequences that area unit extracted from one MODIS statistic transitions.

- The temporal window is intended to control on a subsequence of the statistic to extract data from 2 spectral bands from the MODIS product.

Motivation:

- The previous approach doesn't analyze concerning the thing found. Thus it has to be compelled to notice the new applied mathematics approach.

- Conventional laborious agglomeration strategies classify every purpose of the info set simply to at least one cluster.

- Poor distinction, overlapping intensities and noise scale back the segmentation potency area unit followed during this projected system.

- The projected system doesn't take into account about any abstraction context wherever the activity happens. 


\section{International Journal of Advanced Research in Computer and Communication Engineering}

Vol. 7, Issue 11, November 2018

\section{LITERATURE SURVEY}

J. Hansen et al [1] describe the Goddard Institute for Space Studies (GISS) analysis of global surface temperature change, compare alternative analyses, and address questions about perception and reality of global warming. Satelliteobserved nightlights are used to identify measurement stations located in extreme darkness and adjust temperature trends of urban and peri-urban stations for non-climatic factors, verifying that urban effects on analyzed global change are small. Because the GISS analysis combines available sea surface temperature records with meteorological station measurements, we test alternative choices for the ocean data, showing that global temperature change is sensitive to estimated temperature change in Polar Regions where observations are limited.

Felix N. Kogan et al [2] describe the main goal of global agriculture and the grain sector is to feed 6 billion people. Frequent droughts causing grain shortages, economic disturbances, famine, and losses of life limit the ability to fulfill this goal. To mitigate drought consequences requires a sound early warning system. The National Oceanic and Atmospheric Administration (NOAA) has recently developed a new numerical method of drought detection and impact assessment from the NOAA operational environmental satellites. The method was tested during the past eight years, adjusted based on users' responses, validated against conventional data in 20 countries, including all major agricultural producers, and was accepted as a tool for the diagnosis of grain production. Now, drought can be detected 4-6 weeks earlier than before, outlined more accurately, and the impact on grain reduction can be predicted long in advance of harvest, which is most vital for global food security and trade. This paper addresses all these issues and also discusses ENSO impacts on agriculture.

Jose A. Sobrino et al [3] describe a SPECTRA (Surface Processes and Ecosystem Changes Through Response Analysis) is one of the core candidate missions which is being proposed for implementation in the European Space Agency (ESA) Earth Explorer program of research oriented missions. The scientific objective of the SPECTRA mission is to describe, understand, and model the role of terrestrial vegetation in the global carbon cycle and its response to climate variability under the increasing pressure of human activity. The SPECTRA satellite will embark an optical hyper-spectral payload covering the solar spectral range $(0.4$ to $2.4 \mathrm{~mm})$ and thermal infrared region (10.3 to $12.3 \mathrm{~mm}$ ). This paper is focused on the land surface temperature retrieval from SPECTRA thermal infrared data. In the first part of the paper, generalized single-channel and split-window methods are discussed and compared, showing that single-channel methods provide similar or better results than split-window methods for low atmospheric water vapor content, whereas split-window methods always provide better results for high atmospheric water vapor content. In the second part of the paper, split-window and dual-angle algorithms have been developed for SPECTRA thermal channels.

Zhengming Wan et al [4] describe about a generalized split-window method for retrieving land-surface temperature (LST) from AVHRR and MODIS data. Accurate radiative transfer simulations show that the coefficients in the splitwindow algorithm tor LST must vary with the viewing angle, if we are to achieve a LST accuracy of about $1 \mathrm{~K}$ for the whole scan swath range (USE ${ }^{*}$ from nadir) and for the ranges of surface temperature and atmospheric conditions over land, which are much wider than those over oceans. We obtain these coefficients from regression analysis of radiative transfer simulations, and we analyze sensitivity and error over wide ranges of surface temperature and emissivity and atmospheric water vapor abundance and temperature. Simulations show that when atmospheric water vapor increases and viewing angle is larger than $45^{\circ}$, it is necessary to optimize the split-window method by separating the ranges of the at- mospheric water vapor, lower boundary temperature, and the surface temperature into tractable subranges.

Juan C. Jimenez-Munoz et al [5] describe a algorithms to retrieve land surface temperature from at-sensor and land surface emissivity data. These algorithms have been specified for different thermal sensors on board satellites, i.e., the algorithm used for one thermal sensor (or a combination of thermal sensors) cannot be used for other thermal sensor. The main goal of this paper is to propose a generalized single-channel algorithm that only uses the total atmospheric water vapour content and the channel effective wavelength (assuming that emissivity is known), and can be applied to thermal sensors characterized with a FWHM (Full-Width Half-Maximum) of around $1 \mathrm{~mm}$ actually operative on board satellites. The main advantage of this algorithm compared with the other single channel methods is that in-situ radio soundings or effective mean atmospheric temperature values are not needed, whereas the main advantage of this algorithm compared with split-window and dual-angle methods is that it can be applied to different thermal sensors using the same equation and coefficients.

Bo-Hui Tang, Kun Shao et al [6] calculable and valid the land surface temperature (LST) from thermal-infrared Channels four $(10.8 \mu \mathrm{m})$ and five $(12.0 \mu \mathrm{m})$ of the Visible and Infrared meter (VIRR) aboard the second-generation Chinese polar-orbiting FengYun-3A (FY-3A) weather satellite. The LST, mean emissivity and part water vapour content (WVC) were divided into many tractable sub-ranges with very little overlap to boost the fitting accuracy. The experimental results showed that the basis mean sq. errors (RMSEs) were proportional to the viewing celestial point angles (VZAs) and WVC. The RMSEs were below one.0 K for VZA sub-ranges but three $0^{\circ}$ or for VZA sub-ranges but $60^{\circ}$ and WVC but $3.5 \mathrm{~g} / \mathrm{cm} 2$, given that the land surface emissivities were acknowledged. 


\section{International Journal of Advanced Research in Computer and Communication Engineering}

Vol. 7, Issue 11, November 2018

Bohui Tang et al [7] the estimate of Land Surface Temperature (LST) from the Chinese 1st operational fixed earth science satellite-FengYun-2C (FY-2C) information in 2 thermal infrared channels (IR1,10.3-11.3 $\mu \mathrm{m}$ and IR2, 11.5$12.5 \mu \mathrm{m}$ ), victimisation the Generalized Split-Window (GSW)algorithm projected by Wan and Dozier (1996). The coefficients within the GSW formula cherish a series of overlapping move of the mean emissivity, the atmospherical vapour Content (WVC), and also the LST were derived employing a simple regression method from the numerical values simulated with AN correct atmospherical radiative transfer model MODTRAN four over a good vary of atmospherical and surface conditions

\section{LAND SURFACE TEMPERATURE MODEL}

In this paper work that recognized importance of land surface temperature, correct measurements of LST over continents aren't however accessible for the full globe, for clear and cloudy skies, with a time sampling adequate resolve the diurnal cycle and to research synoptic, seasonal, and inter-annual variability. Accurate radiative transfer simulations show that the coefficients of this LST rule depends on viewing angle, to attain a LST accuracy of concerning one $\mathrm{K}$ for the full scan swath vary and for the ranges of surface temperature and region conditions over land, that area unit abundant wider than those over oceans. They acquire these coefficients from multivariate analysis of radiative transfer simulations, and analyze sensitivity and error over wide ranges of surface temperature and emissivity and region water vapour abundance and temperature. The region lower boundary temperature and (vertical) column water vapour values retrieved from MODIS region sounding channels will be accustomed confirm the vary for the optimum coefficients of the split-window methodology. This viewing-angle dependent rule not solely retrieves LST a lot of accurately, however it's conjointly less sensitive than viewing-angle freelance LST algorithms to the uncertainty within the band emissivities of the land-surface within the split-window and to the instrument noise. The major problem in victimisation this generalized split-window LST rule is a way to assign acceptable band emissivities for every component in real process. it's necessary to reinforce the emissivity knowledge domain of natural terrestrial materials and to develop new algorithms for at the same time retrieving surface emissivities and temperature for land covers with variable emissivities.

- Low-resolution sensors.

- Multispectral/hyper spectral thermal information.

- The supervised approach should even be strong to errors occurring at intervals the coaching set.

- The existing system doesn't provide the applied mathematics information like variety of objects found.

- Conventional laborious agglomeration strategies classify every purpose of the info set simply to at least one cluster.

- Poor distinction, overlapping intensities and noise scale back the segmentation potency.

- The projected approach doesn't take into account about any abstraction context.

- The result provided restricted abstraction resolution, poor distinction, overlapping intensities.

- There is not any thanks to management the trade-off between smoothing and agglomeration

The surface temperature in international and regional models is crucially vital attributable to relevancy to the computations of the turbulent heat fluxes also because the terrestrial radiation. Remotely detected land surface temperature information area unit thought-about to contain valuable data on the presence and nature of vegetation, heat fluxes, and also the wetness convenience. The projected model is forced to match the ascertained part temperatures by dynamic each the soil wetness content and also the water roughness for the warmth exchange of the vacant ground part. In this projected model is thesis temperature vertically and whereas the satellite observations area unit angle dependent. Satellites observe real surfaces whereas heterogeneousness is parameterized in models (if at all). These inconsistencies add uncertainty to the comparison of model and remotely detected LST for information assimilation functions. Whereas some inconsistencies area unit possible to not be eliminated fully, information assimilation should account for them as a matter of observe.

The distinction in modification detection accuracy between the $\$ 64000$ and simulated land cowl action was still tolerably little in these experiments, although solely a restricted variety of real land conversion examples were accessible. Clustering techniques area unit broadly speaking divided into class-conscious and partitional agglomeration approaches. The Fuzzy Automatic agglomeration rule is associate degree clustered class-conscious agglomeration methodology that produces a nested hierarchy of clusters of distinct objects in step with some reasonably proximity matrix. The partitional agglomeration methodology creates associate degree unnested partitioning of the info points with clusters. A silhouette graph was accustomed confirm the optimum variety of clusters for partitional agglomeration and resulted in two clusters being the simplest selection LST.

- An correct region correction isn't required.

- The physical land surface detection will be applied to sensors with any MIR band or even to sensors while not MIR bands information.

- The projected methodology provides surface temperature and emissivity, synchronic analysis. 
- The correct region correction agglomeration model is projected.

- The image classification between pixels of low or high spectral distinction, that causes

- Art factual discontinuities on the emissivity merchandise.

- The totally different agglomeration techniques area unit used for image analysis even in dynamic atmospheric condition over time.

- The projected system provides the applied mathematics information like variety of objects found and share of space coated.

\section{FUZZY RANDOM CLUSTERING MODEL}

A. Image Notification Settings: In this module, the date and time of image taken is keyed in and the land image is selected. The image format may be bitmap, JPEG, GIF, TIFF or any other format. Normally satellite images are selected. The details are saved in the database as well as the image is saved in 'land images' folder. These are used in further modules. The collection of images can also be selected and the time interval is given so that more number of records can be saved at a given time.

\section{B. Clustering with Segmentation for Regular Interval}

i) Configure Cluster Values: The main form's tab control's first tab page is having options for selecting cluster count, iteration count and precision. Three numeric up down controls are provided to select the count. For sake of convenience, cluster count range is from two to ten; iteration count range is from one to twenty and precision is 0.005 to 1 with increment value 0.05 .

ii) Applying Fuzzy Automatic Clustering Algorithm: The main form's tab control's second tab page is having option for applying Fuzzy Automatic Clustering Algorithm with new fuzzy factor. During the processing, the values for cluster, weight i.e., fuzziness factor set to value two, and epsilon value 10 to the power of -5 are set. Then the fuzzy partition matrix is initialized. Then cluster centers are calculated along with membership matrix. The steps are iterated for given number of times to segment the image further.

C) Identification of Land-Cover Change: In this module, the images are collected in chronological order. Then the clustered area (i.e., the land area (marked in color like brown)) is retrieved with their location information. The $\mathrm{X}$ and $\mathrm{Y}$ coordinates of each and every pixel marked as land are saved for all images. Then the land change i.e., pixel color modified in the respective area are calculated. If the number of pixels calculated are above the given threshold value, then the land segment is considered as changed. The land changed area is converted to gray scale segment so that it can be identified easily.

D) Identification and Change Detection Over Time: In this module, the images are collected in chronological order. Then the animal image location (marked as a given irregular shape)) is retrieved with their location information. The $\mathrm{X}$ and $\mathrm{Y}$ coordinates of each and every pixel marked as animal are saved for all images. Then the animal position change i.e., pixel color modified in the respective area are calculated. The animal located area is converted to gray scale segment so it can be identified easily.

E) Image Filter Process: In the form, the noise in the image is filtered by changing the pixel value with median values of surrounding pixels. To apply median filter, for each pixel, the surrounding pixels $3 \times 3$ is taken and the gray scale values are su mmed and median value is found out. The median value is set to the center pixel. This reduces the noise data in segmented image for clear view of output image.

F) Statistical Data Model: In this module, the after the image segmentation, the number of objects such as water, thin grass land, thick grass land or the animals found are calculated. In addition, the percentage occupied by the corresponding elements are also calculated and displayed. This assists in percentage of land change with respect to previous images.

G) Land Changed Status Update Service: In this module, farmers land location is saved with address information. If the address is keyed in, the latitude and longitude information can be retrieved and saved. During the image addition, the latitude and longitude information is also saved. When the land status is changed, then the owner of the land is retrieved and is intimated with the statistics through 'sms'.

H) Integration of Modules: The thesis start with the admin module once the login is made the control moves to the main page. This page contains image, time frame, people registration, detections and the view for the entire previously added document. Add the land image that is collected from the satellite and then that is stored into the database the stored image is mentioned with specific time interval. The image collected is stored using the picture box control and different textbox and the listbox controls. The command click is made for save and the exit option in the page. After that the control move on to the segmentation form in this form image added is segmented for that it will take different 
iteration and the segmentation is to be done within that iteration count and the precision count are also taken as the input and by using the list box control the iteration value is set after the command click next is clicked the control will move on to the next page. The next is that segmentation form when $\mathrm{c}$ means is applied to the selected image in this form. The command click median filter is clicked the image filtration is happened on the selected image and image output will be produced in this form. After the segmentation is completed the message window is displayed that segmentation is completed successfully and the output is shown in the picture box.

After this the control moves to the intermediate image form in this the intermediate image is filtered and the output will be produced in this form. The next form is time frame setting form in this form the time duration is set for the taking the snap image through satellite is done in this form. The next form is the people registration in this form the details about the people living area and their land type like details are stored in this form. This form also includes short message setup. The message will be send to the people in the critical region after the command click is made. The next form in this thesis is detection in this form the source image is selected and the after the filter is applied on that image. The next form is view in this different view is generated to the previously added details in this form. This includes land area changes and the location changes are done in this form. Finally the different view is generated for the location and land area changes.Land surface emissivity (LSE). Average emissivity of a part of the surface of the planet calculated from measured radiance and land surface temperature (LST).

- Atmospheric window. A spectral wavelength region during which the atmosphere is sort of clear, separated by wavelengths at that region gases absorb radiation.

- Blackbody. a perfect material fascinating all incident energy or emitting all thermal energy attainable. A cavity with a puncture aperture approximates a full radiator.

- Brightness temperature. The temperature of a full radiator that will offer the radiance measured for a surface.

- Color temperature. Temperature satisfying Planck's law for spectral radiances measured at 2 completely different wavelengths.

- Contrast stretch. Mathematical remodel that adjusts the method during which nonheritable radiance knowledge translate to the black/white dynamic vary of the show monitor.

- Emissivity e. The potency with that a surface radiates its thermal energy.

- Irradiance. the ability incident on a unit space, integrated over all directions (W m2).

- Gray body. a cloth having constant however non-unity emissivity

\section{PERFORMANCE ANALYSIS}

This table 5.1 shows the details of the image that is image taken from different locations or different area the size of the image in mega byte. The existing analysis and the proposed fuzzy automatic clustering are listed in this table.

Table 5.1 Area Wise Analysis

\begin{tabular}{|l|c|c|c|}
\hline Area wise image & Size of image in MB & Existing analysis & Fuzzy automatic clustering \\
\hline Water area & 55 & 23 & 34 \\
\hline Land area & 62 & 30 & 40 \\
\hline Agriculture area & 74 & 42 & 50 \\
\hline Industrial area & 82 & 52 & 63 \\
\hline Populated area & 95 & 66 & 78 \\
\hline
\end{tabular}

The below Fig 5.1 shows that area wise analysis that is existing analysis and the fuzzy automatic clustering are shown in this chart.

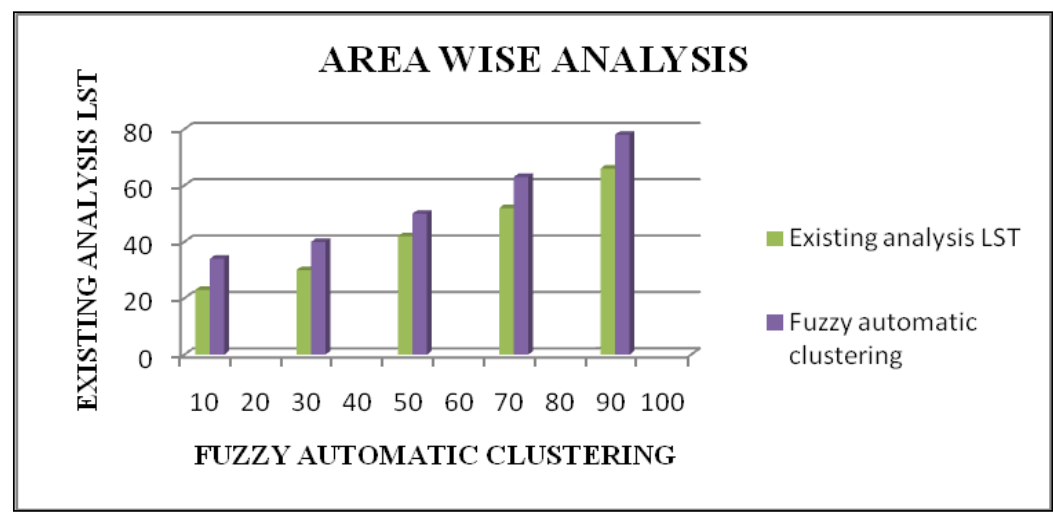

Fig 5.1 Average Analysis 
Vol. 7, Issue 11, November 2018

This table 5.2 shows the details of the image that is image taken from time to time that are represented in milliseconds. The existing analysis and the proposed fuzzy automatic clustering are listed in this table with their time analysis.

Table 5.2 Time Analysis

\begin{tabular}{|l|c|c|c|}
\hline Area wise image & $\begin{array}{c}\text { Size of image } \\
\text { in MB }\end{array}$ & Existing analysis in m sec & $\begin{array}{c}\text { Fuzzy automatic clustering } \\
\text { in } \mathbf{~ m} \text { sec }\end{array}$ \\
\hline Water area & 55 & 0.011 & 0.009 \\
\hline Land area & 62 & 0.026 & 0.019 \\
\hline Agriculture area & 74 & 0.035 & 0.022 \\
\hline Industrial area & 82 & 0.046 & 0.036 \\
\hline Populated area & 95 & 0.054 & 0.044 \\
\hline
\end{tabular}

The below Fig 5.2 shows that time wise analysis that is existing analysis and the fuzzy automatic clustering are shown in this chart.

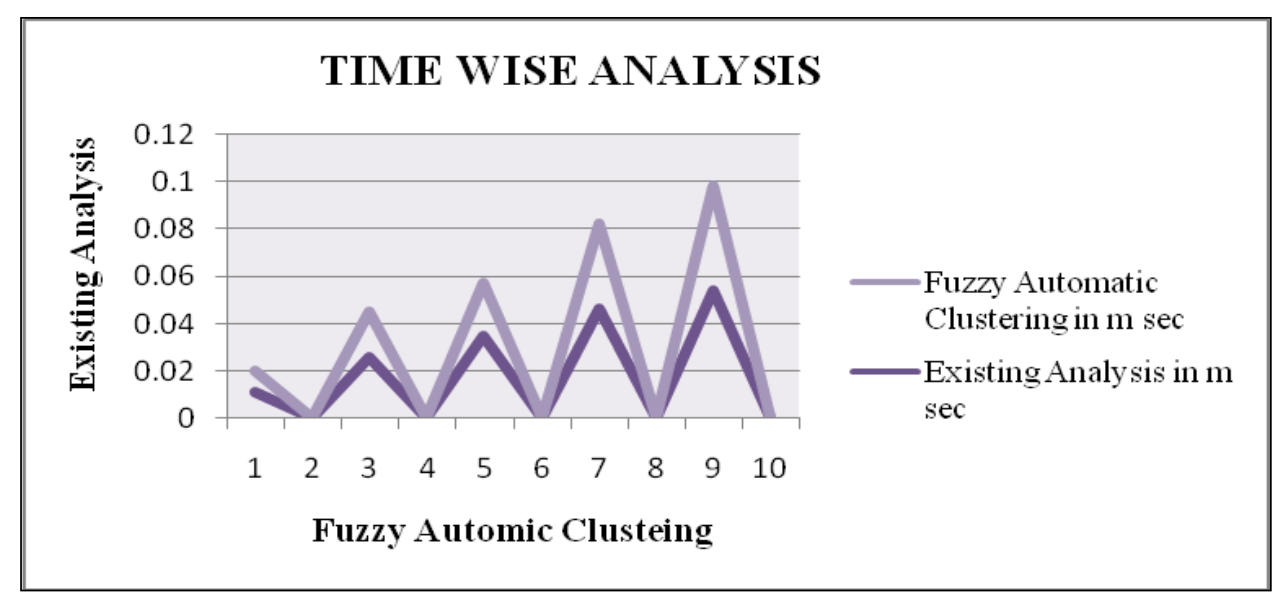

Fig 5.2 Time Wise Analysis

\section{CONCLUSION}

In this projected system the Lane Surface Area and also the water space regions are separated victimization the MODIS Satellite knowledge the particular space image is been chosen and also the temperature in this area is been known. The previous study doesn't concentrate within the specific region they contemplate the whole region so the accuracy of the estimation are the main drawback to find the temperature. The projected system concentrate to find the desired space drawback by victimisation the MODIS image in this a section of the place is chosen which is separated because the land and also the water area and also the temperature is been noted. On the premise of radiative transfer theory within the MIR region, a bi-face reflectivity retrieval methodology was wont to separate the mirrored star direct irradiance and also the radiances emitted by the surface and atmosphere. A kernel-driven model was projected to explain the non-Lambertian reflective behavior of the land surface and to consequently confirm the directional emissivity if there have been quite 3 phase reflectance out there with totally different angular configurations on many consecutive days. The results showed that the bias and RMSE between the LSTs retrieved from MODIS daytime MIR knowledge and people calculated victimisation in place measurements, at the time of the MODIS pictures. The projected methodology may be wont to accurately retrieve LST from MODIS daytime MIR knowledge.

\section{FUTURE ENHANCEMENT}

The projected system solely concentrates to find the changes within the agricultural space solely however this could be taken for the thought. That's the Lane surface area changes are happened within the wild life Surface area and additionally within the beach areas. This projected system is extended within the all the regions to intimate the land temperature variations. It's additionally includes mountains and also the hill stations concerning sand slope like nature disasters to the nearer individuals. This identification period is altered and also the satellite image taken can even be altered during this future arrange. Rather than taking the image to find the changes the video that's recorded within the satellite could taken for the analysis. 


\title{
International Journal of Advanced Research in Computer and Communication Engineering
}

\author{
Vol. 7, Issue 11, November 2018
}

\section{REFERENCES}

[1]. J. Hansen, R. Ruedy, M. Sato, and K. Lo, “Global surface temperature change,’Rev. Geophys., vol. 48, no. 4, Dec. 2010 , Art. no. RG4004.

[2]. F. N. Kogan, “Operational space technology for global vegetation assessment,”Bull. 3 Amer. Meteorol. Soc., vol. 82, no. 9, pp. 19491964, Sep. 2001.

[3]. J. A. Sobrino and J. C. Jiménez-Muñoz, "Land surface temperature retrieval from thermal infrared data: An assessment in the context of the surface processes and ecosystem changes through response analysis (SPECTRA) mission,” J. Geophys. Res., vol. 110, no. D16, Aug. 2005, Art. no. D16103.

[4]. Z. Wan and J. Dozier, "A generalized split-window algorithm for retrieving land-surface temperature from space," IEEE Trans. Geosci. Remote Sens., vol. 34, no. 4, pp. 892-905, Jul. 1996.

[5]. J. C. Jiménez-Muñoz and J. A. Sobrino, "A generalized single-channel method for retrieving land surface temperature from remote sensing data,” J. Geophys. Res., vol. 08, no. D22, pp. 4688-4695, Nov. 2003.

[6]. B.-H. Tang et al., "Estimation and validation of land surface temperature from Chinese second generation polar-orbiting FY-3A VIRR data," Remote Sens., vol. 7, no. 3, pp. 3250-3273, Mar. 2015.

[7]. C. Gao, B.-H. Tang, H. Wu, X. Jiang, and Z.-L. Li, "A generalized split window algorithm for land surface temperature estimation from MSG-2/SEVIRI data,” Int. J. Remote Sens., vol. 34, no. 12, pp. 4182-4199,Jun. 2013.

[8]. A. Berk et al., "MODTRAN cloud and multiple scattering upgrades with application to AVHRIS,'Remote Sens. Environ., vol. 65, no. 3, pp. 367-375, Sep. 1998.

[9]. Eduardo de Miguel, Elena Prado, Marcos Jiménez, Cristina Robles, "Evaluating the Information Content of the AHS MIR Bands", Proceedings $5^{\text {th }}$ EARSeL Workshop on Imaging Spectroscopy. Bruges, Belgium, April 23-25 2007.

[10]. Alan Gillespie, "Land Surface Emissivity", Encyclopedia of Remote Sensing, DOI 10.1007/978-0-387-36699-9, Springer Science+Business Media New York 2014

[11]. Lang Xia Kebiao Mao, Ying Ma, Fen Zhao, “An Algorithm for Retrieving Land Surface Temperatures Using VIIRS Data in Combination with Multi-Sensors", Sensors 2014, 14, 21385-21408; doi:10.3390/s141121385.

[12]. José A. Sobrino, Juan C et al, "Land Surface Emissivity Retrieval From Different VNIR and TIR Sensors", IEEE Transactions on Geo-Science and Remote Sensing, Vol. 46, NO. 2, February 2008.

[13]. Li Fang, Yunyue Yu, Hui Xu, and Donglian Sun, "New Retrieval Algorithm for Deriving Land Surface Temperature from Geostationary Orbiting Satellite Observations", IEEE Transactions on Geo-Science and Remote Sensing, Vol. 52, No. 2, February 2014.

[14]. Xiaolei Yu, Xulin Guo and Zhaocong Wu, "Land Surface Temperature Retrieval from Landsat 8 TIRS-Comparison between Radiative Transfer Equation-Based Method, Split Window Algorithm and Single Channel Method“,Remote Sens. 2014, 6, 9829- 9852; doi:10.3390/rs6109829.

[15]. Ying Sun, "Retrieval and Application of Land Surface Temperature", - geo.utexas.edu , Term Paper, 2011.

[16]. Zhao-Liang Li, Ronglin Tang et al, "A Review of Current Methodologies for Regional Evapotranspiration Estimation from Remotely Sensed Data", Sensors 2009, 9, 3801- 3853.

[17]. J. Hansen, R. Ruedy, M. Sato, and K. Lo, “Global surface temperature change,”Rev. Geophys., vol. 48, no. 4, Dec. 2010, Art. no. RG4004.

[18]. F. Sattari and M. Hashim, "A Brief Review of Land Surface Temperature Retrieval Methods from Thermal Satellite Sensors", Middle-East Journal of Scientific Research 22 (5): 757-768, 2014.

[19]. Zhengming Wan, "Validation and Refinement of the MODIS Land-Surface Temperature Product", DIS Science Team Meeting, May 18-20, 2011.

[20]. Guillem Sòria, José A. Sobrino, Mariam Atitar et al, "AATSR Land Surface Temperature Product: Comparison With SEVIRI and MODIS In The Framework Of CEFLES2 Campaigns”, Proc. of the '2nd MERIS/ (A)ATSR User Workshop', Frascati, Italy 22-26September 2008. 